This is the pre-peer reviewed version of the following article:

W. P. du Plessis, "Interdigital Filter Design," Microwave and Optical

Technology Letters, vol. 51, no. 10, pp. 2269-2272, October 2009,

which has been published in final form at:

http://www3.interscience.wiley.com/journal/122521236/abstract 


\title{
Interdigital Filter Design
}

\author{
Warren P. du Plessis \\ Affiliation: Council for Scientific and Industrial Research (CSIR) \\ Postal Address: PO Box 395, Pretoria, 0001, South Africa \\ E-mail: wduplessis@csir.co.za \\ Fax: +27 12 841-2455
}

\begin{abstract}
A new synthesis procedure for interdigital filters with shorted-pin feeds is developed by relating the coupling factors and external Qs to the physical structure of the filter. This new procedure is easily understood and applied, extremely flexible, and allows design tradeoffs to be evaluated in an intuitive way.
\end{abstract}

Keywords: Cavity resonator filters, microwave filters, coupled transmission lines.

\section{Introduction}

Interdigital filters are popular at the higher microwave frequencies for a number of reasons. Ideal interdigital filters have perfect symmetry which means that they have better phase and delay characteristics than combline filters [1]. The couplings between the resonators of interdigital filters are also lower than those of equivalent combline filters. This means that the gaps between resonators in an interdigital filter are larger, easing construction at higher frequencies [1]. The two main techniques for designing interdigital filters are those developed by Matthaei [2] and Wenzel [3].

Matthaei's design approach [2] uses an approximation to the response of an ideal interdigital filter that has proved to be surprisingly accurate, and can be utilised for bandwidths of up to an octave [4]. The greatest advantages of Matthaei's design process are that it is easy to use and it 
produces good results. Unfortunately, Matthaei's approach is extremely rigid and limits the designer's freedom to adjust filter dimensions.

Wenzel's synthesis procedure [3] is based on capacitance matrices, and has the benefits of being exact and extremely flexible. This flexibility can be seen by the number of unusual physical realisations proposed in Wenzel's paper [3]. However, the price of these benefits is that the procedure involves complicated matrix manipulations.

A further drawback of both these design procedures is that they do not lend themselves to gaining insight into the effect of system tradeoffs. Matthaei's work presents a set of equations that offer very little scope for variation, while Wenzel's approach limits insight by being complex.

Equations relating the coupling factors and external Qs of interdigital filters to the structure capacitances are described below. These equations lead directly to an uncomplicated, intuitive and flexible design procedure. Furthermore, these equations aid visualisation of the relationship between the physical structure of an interdigital filter, and its coupling factors and external Qs.

Section 2 presents the derivation of equations for the coupling factors and external Qs. These equations are then used as the basis for a design procedure described in Section 3, which is used to design a filter in Section 4. Finally, the benefits of this new approach are discussed in Section 5, and a brief conclusion is given in Section 6.

\section{Derivation}

The derivation of equations for the coupling factors and external Qs starts with the well-known transmission-line model of a shorted-pin feed interdigital filter shown in Figure 1 [5]. An ideal transformer with a turns ratio of 1:-1 should be inserted at one end of the filter in Figure 1 when the filter consists of an even number of resonators. However, this transformer has no effect on the calculations below and is thus ignored.

Figure 1 can be modified to the general inverter-coupled resonator bandpass filter structure in Figure 2 [6] by using the inverter shown in Figure 3. The admittance of the inverter in Figure 3 is 


$$
J=\frac{Y_{m n}}{\sin (\beta l)}
$$

where $\beta$ is the propagation factor and $l$ is the length of the resonators (a quarter wavelength in this case). While the series quarter-wave line alone can approximate an inverter, the structure shown in Figure 3 is more accurate over wide bandwidths.

At this point the circuits in Figures 1 and 2 still differ because the feed pins are not present in Figure 2. The feed pins can be removed by setting $Y_{0}=1 / R$ where $R$ is the load impedance value, and modifying the value of $Y_{1}$ to be [6]

$$
Y_{1 \text { mod }}=Y_{1}-\frac{Y_{01}^{2}}{Y_{0}} .
$$

The coupling factor of two parallel resonators coupled by an inverter is given by [6]

$$
k_{m n}=\frac{J_{m n}}{\sqrt{b_{m} b_{n}}}
$$

where $J_{m n}$ is the inverter admittance, and the $b$ values are given by

$$
b=\left.\frac{\omega_{0}}{2} \cdot \frac{\partial B}{\partial \omega}\right|_{\omega=\omega_{0}}
$$

where $\omega$ is the frequency and $\omega_{0}$ is the resonant frequency. The admittance $B$ above is the susceptance of the resonator and must have a value of zero at resonance for (4) to be valid. The input susceptance of a shorted transmission line is

$$
B_{n}=\frac{-Y_{n}}{\tan (\beta l)}
$$

Substituting (5) into (4) and simplifying gives

$$
b_{n}=\frac{\pi}{4} Y_{n}
$$

This result can then be substituted in (3) to give

$$
k_{m n}=\frac{4}{\pi} \cdot \frac{Y_{m n}}{\sqrt{Y_{m} Y}} .
$$


The coupling factor between two pins thus depends on the admittances of the two transmission lines and the admittance of the inverter between them. The values of $Y_{1 \text { mod }}$ given in (2) should be used in (7) instead of $Y_{1}$ for the first resonators.

The external Qs of a filter are defined as [6]

$$
Q_{E}=\frac{b_{1}}{J_{01}^{2} R}
$$

where $b_{1}$ is calculated using the modified value of $Y_{1}$ given in (2) to give

$$
b_{1}=\frac{\pi}{4}\left(Y_{1}-\frac{Y_{01}^{2}}{Y_{0}}\right)
$$

Substituting this value into (8) and simplifying gives

$$
Q_{E}=\frac{\pi}{4}\left(\frac{Y_{1}}{Y_{01}^{2}} \frac{1}{R}-1\right)
$$

The external Qs thus depend on the admittance of the first resonator, the admittance of the first inverter and the load impedance. The feed pin admittance does not directly affect the external Qs, but must be set equal to $1 / R$ as described above.

Since the TEM assumption is assumed valid, the admittances of the transmission lines in Figure 1 are related to their static capacitances by

$$
Y=v C
$$

where $Y$ and $C$ are the admittance and capacitance of the transmission line respectively, and $v$ is the speed of light. The capacitance of a resonator is the capacitance of the resonator transmission line with all the other transmission lines shorted to ground. The capacitance associated with an inverter is half the difference between the odd- and even-mode capacitances of the two transmission lines coupled by the inverter.

The admittances in (7) and (10) can be converted to capacitances using (11) to give

$$
k_{m n}=\frac{4}{\pi} \cdot \frac{C_{m n}}{\sqrt{C_{m} C_{n}}}
$$




$$
Q_{E}=\frac{\pi}{4}\left(\frac{C_{1}}{v C_{01}^{2}} \cdot \frac{1}{R}-1\right)
$$

These forms are more intuitive than (7) and (10) because the relationships between the dimensions of a filter and its capacitances are readily visualised.

Two approximations were made in the course of this derivation. The first is that the inverter shown in Figure 3 is ideal. The second approximation is that the coupling factors and external Qs calculated at resonance are valid over wide bandwidths. These approximations are the same as those made by Matthaei [2], so the results should be valid over similarly wide bandwidths of up to an octave [4].

\section{Design Procedure}

The equations derived in Section 2 will now be used to formulate a design procedure for interdigital filters with shorted-pin feeds.

The values of the desired coupling factors and external Qs can be found from $[6,7]$

$$
k_{m n}=\frac{\Delta \omega}{\omega_{0} \sqrt{g_{m} g_{n}}}
$$

and

$$
Q_{E}=\frac{g_{0} g_{1} \omega_{0}}{\Delta \omega}
$$

where $\Delta \omega$ is the bandwidth, and the values of $g_{n}$ are the component values of a conventional normalised low-pass filter prototype. The $g_{n}$ values can either be calculated or obtained from standard filter tables [6].

Once the desired values of the filter parameters are known, (7) and (10) or (12) and (13) can be used to realise these values. The values of the capacitances can be determined from graphs and tables such as those provided in [6] or from electrostatic simulators. 


\section{Example}

The design procedure described in Section 3 will be demonstrated by designing a filter with the specifications given in Table 1. The example considered here is taken from Matthaei [2] to allow comparisons to be made.

The filter prototype values obtained from [6], and the coupling factors and external Qs calculated from (14) and (15) are given in Table 2. The inherent symmetry of interdigital filters is clearly shown by the fact that the coupling coefficient and external $Q$ values in Table 2 are symmetrical despite the fact that the prototype filter coefficients are not.

Matthaei chooses his sole design parameter so that resonator impedances are close to $76 \Omega$ to obtain optimum resonator Q. The values used here were recalculated using Matthaei's equations and differ slightly from the results published in [2] due to rounding errors.

The impedances of all the resonators, except the first, were set to $76 \Omega$ to allow comparisons with Matthaei's results. The modified admittance $\left(Y_{1 \text { mod }}\right)$ was set to $1 / 76 \Omega$ by adjusting admittance of the first resonator $\left(Y_{1}\right)$ to better match the approach followed by Matthaei. The coupling capacitances were then calculated from (12) and (13).

The gap between the feed pin and the first resonator is the smallest, and manufacturing difficulties can be encountered when this gap becomes too small, so the size of this gap was increased by decreasing its admittance. This change was compensated for by adjusting the admittance of the first resonator and the admittance between the first two resonators.

Table 3 shows that the admittances in Matthaei's design and the comparable design using the current method are essentially identical. The agreement between the frequency responses given in Figure 4 is so good that it is virtually impossible to see the differences between the curves. 


\section{Discussion}

The design procedure described in Section 3 is uncomplicated, intuitive and flexible as demonstrated in Section 4. The flexibility arises because the designer is free to modify the structure and all the available dimensions as long as the correct coupling factors and external Qs are realised.

The simple form of equations (7), (10), (12) and (13) facilitates insight during the design procedure. For example, (12) shows that the gap between two resonator pins can be increased (decreasing the capacitance between the pins) as long as the width of one or both the pins is decreased to compensate (by decreasing the resonator capacitance).

This type of insight is particularly valuable when tuning filters. The external Qs and coupling factors can be measured using the procedures described by Ness [7], and any deviations from the desired values can then be removed by tuning the dimensions of the filter. The equations derived in Section 2 allow the designer to evaluate which of the possible modifications to make, and what the effect of those modifications on the filter dimensions will be.

\section{Conclusion}

Equations for the coupling factors and external Qs of interdigital filters with shorted-pin feeds have been derived. These equations lead naturally to a design procedure that is less complicated and more flexible than other current approaches. The simple form of the equations allows insight into the effect of changes to the filter structure to be gained. 


\section{References}

[1] R. Levy, R. V. Snyder, and G. L. Matthaei, Design of microwave filters, IEEE Trans. MTT 50 (2002), 783-793.

[2] G. L. Matthaei, Interdigital band-pass filters, IRE Trans. MTT 10 (1962), 479-491.

[3] R. J. Wenzel, Exact theory of interdigital band-pass filters and related coupled structures, IEEE Trans. MTT 13 (1965), 559-575.

[4] R. Levy and S. B. Cohn, A history of microwave filter research, design, and development, IEEE Trans. MTT, Vol. 32 (1984), 1055-1067.

[5] R. Sato and E. G. Cristal, Simplified analysis of coupled transmission-line network, IEEE Trans. MTT, Vol. 18 (1970), 122-131.

[6] G. L. Matthaei, L. Young, and E. M. T. Jones, Microwave filters, impedance-matching networks and coupling structures, Artech House, Dedham, 1980.

[7] J. B. Ness, A unified approach to the design, measurement and tuning of coupled-resonator filters, IEEE Trans. MTT, Vol. 46 (1998), 343-351. 


\section{Figures}

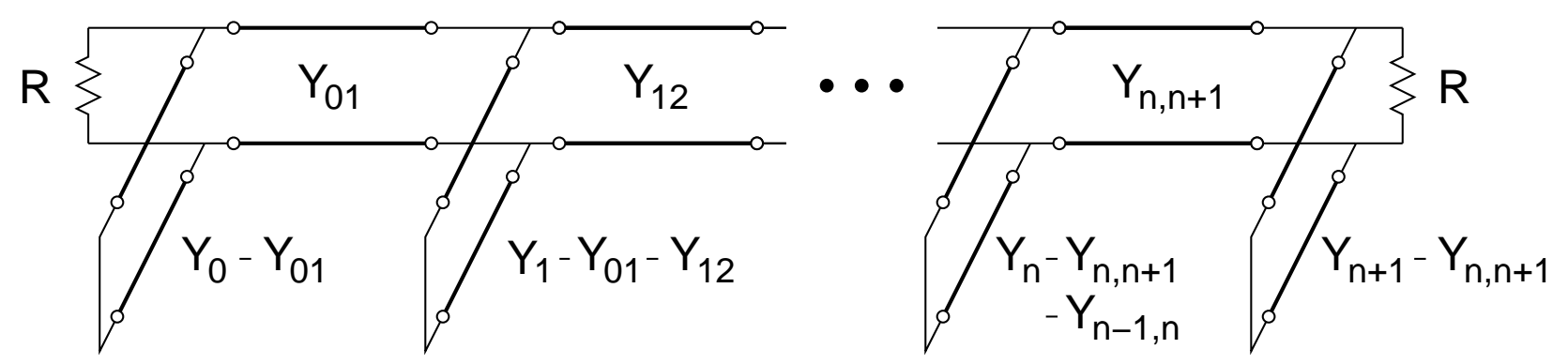

Figure 1: Interdigital filter transmission-line equivalent circuit. All transmission lines are a quarter wavelength long.

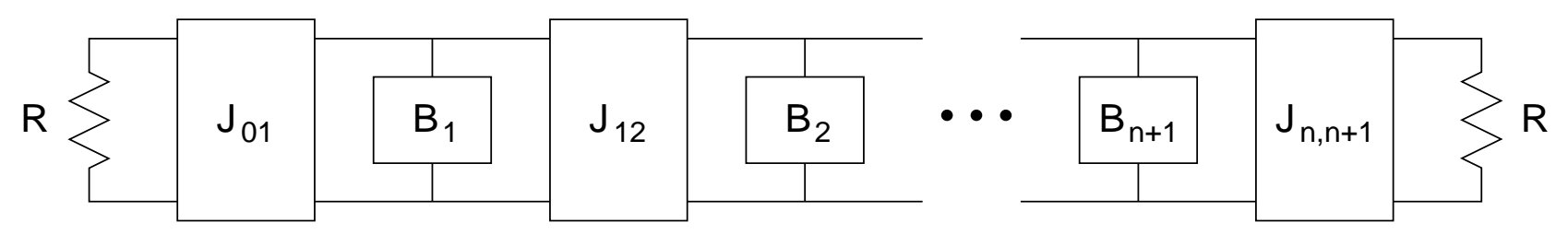

Figure 2: Ideal inverter-coupled resonator bandpass filter.

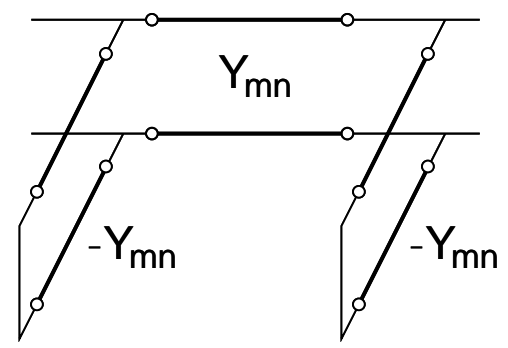

Figure 3: Inverter model. 


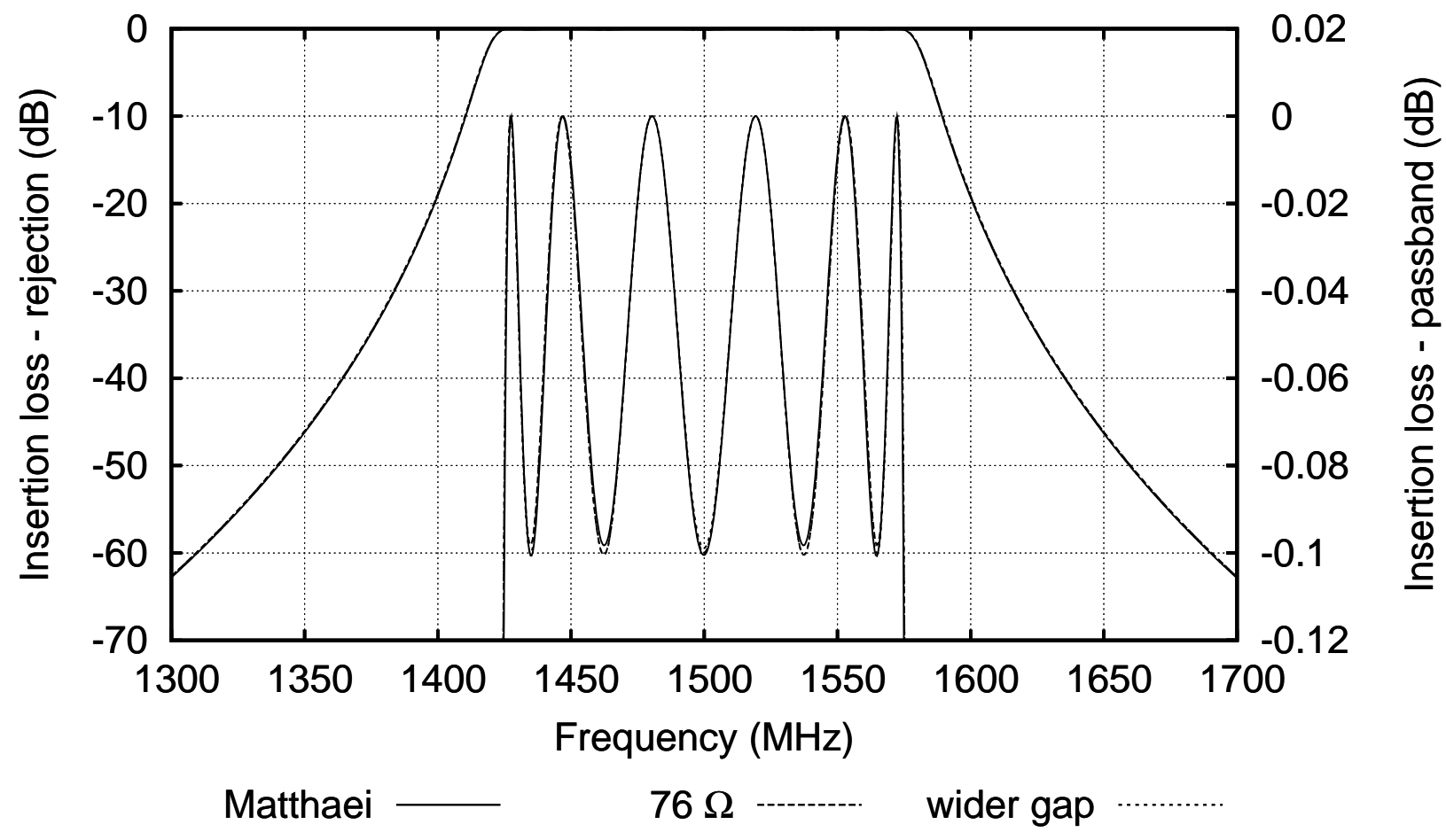

Figure 4: Filter frequency response. 
Table Headings

Table 1: Example filter design parameters [2].

\begin{tabular}{|l|c|}
\hline Parameter & Value \\
\hline Order (number of resonators) & 6 \\
Centre frequency & $1.5 \mathrm{GHz}$ \\
Bandwidth & $150 \mathrm{MHz}$ \\
Passband ripple & $0.1 \mathrm{~dB}$ \\
\hline
\end{tabular}

Table 2: Prototype and calculated filter values.

\begin{tabular}{|c|c|c|c|}
\hline Index & $\begin{array}{c}\text { Prototype } \\
\text { value }\left(g_{n}\right)\end{array}$ & $\begin{array}{c}\text { Coupling } \\
\text { factor }\left(k_{m n}\right)\end{array}$ & $\begin{array}{c}\text { External Q } \\
\left(Q_{E}\right)\end{array}$ \\
\hline 0 & 1.0000 & & 11.68 \\
1 & 1.1681 & 0.07809 & \\
2 & 1.4039 & 0.05886 & \\
3 & 2.0562 & 0.05662 & \\
4 & 1.5170 & 0.05882 & \\
5 & 1.9029 & 0.07809 & \\
6 & 0.8618 & & 11.68 \\
7 & 1.3554 & & \\
\hline
\end{tabular}

Table 3: Normalised capacitances for filter designs.

\begin{tabular}{|c|c|c|c|}
\hline Index & Matthaei $[2]$ & $76 \Omega$ resonators & Wider gap \\
\hline 0 & 20.00 & 20.00 & 20.00 \\
01 & 4.197 & 4.206 & 3.000 \\
1 & 14.00 & 14.04 & 7.143 \\
12 & 0.803 & 0.807 & 0.576 \\
2 & 13.15 & 13.16 & 13.16 \\
23 & 0.605 & 0.608 & 0.608 \\
3 & 13.12 & 13.16 & 13.16 \\
34 & 0.582 & 0.585 & 0.585 \\
4 & 13.12 & 13.16 & 13.16 \\
45 & 0.605 & 0.608 & 0.608 \\
5 & 13.15 & 13.16 & 13.16 \\
56 & 0.803 & 0.807 & 0.576 \\
6 & 14.00 & 14.04 & 7.143 \\
67 & 4.197 & 4.206 & 3.000 \\
7 & 20.00 & 20.00 & 20.00 \\
\hline
\end{tabular}

